\title{
STABILITY ANALYSIS OF STOCHASTIC RICKER POPULATION MODEL
}

\author{
NATALI HRITONENKO, ALEXANDRA RODKINA, AND YURI YATSENKO
}

Received 10 October 2005; Accepted 19 December 2005

A stochastic generalization of the Ricker discrete population model is studied under the assumption that noise impacts the population reproduction rate. The obtained results demonstrate that the demographic-type stochastic noise increases the risk of the population extinction. In particular, the paper establishes conditions on the noise intensity under which the population will extinct even if the corresponding population with no noise survives.

Copyright (c) 2006 Natali Hritonenko et al. This is an open access article distributed under the Creative Commons Attribution License, which permits unrestricted use, distribution, and reproduction in any medium, provided the original work is properly cited.

\section{Introduction}

The stability of stochastic difference equations has been investigated in numerous papers (see, e.g., [8, 13-19]). In this paper a martingale-based technique [13] is applied to a stochastic version of the well-known Ricker population model [12]:

$$
x_{n+1}=a e^{-b x_{n}} x_{n}, \quad n=0,1,2, \ldots
$$

The Ricker model (1.1) provides a classic description of an isolated single-species population in the inhibiting environment, which only produces offspring at a specific time each year. It is very popular in biological literature because of its remarkable dynamics and good correspondence with various experimental data (especially, for fish populations) $[4,11]$. As shown in [6], a nonlinear integral population model with distributed delay and intra-species competition can be reduced to model (1.1) in the case of a singletime seasonal reproduction. It is well known that when the parameter $a$ increases from 0 , the qualitative behavior of model (1.1) changes from a single zero stationary state through a stable nonzero stationary state to oscillations (stable cycles) with increasing periods, instability, and quasi-chaotic dynamics (see, e.g., [11] and the references therein). 
While the Ricker model is interesting by itself, there is a number of its stochastic versions where one of the parameters $a$ and $b$ (or both) is of stochastic nature (see [1$3,7,10]$, and others). As noticed in [1], the stochasticity of the parameter $a$ reflects the internal demographic factors of a population whereas the variation of the parameter $b$ represents the effect of the natural environment. Paper [10] establishes conditions of growth dynamics in a stochastic Ricker model. The stability (including ergodicity) of the Ricker model with environmental stochasticity has been explored in [1-3], using the theory of discrete-time Markov chains.

In this paper, we apply the martingale theory to the following generalization of the Ricker model (1.1) with demographic stochasticity:

$$
x_{n+1}=x_{n} e^{-b x_{n}}\left(a_{n}+\sigma_{n} \xi_{n+1}\right), \quad n=0,1,2, \ldots,
$$

where $\xi_{n+1}$ are independent random variables such that $\mathbb{E} \xi_{n+1}=0$ and $\mathbb{E} \xi_{n+1}^{2}=\eta_{n+1}$. We assume that $b>0$ is nonrandom and the following inequality is almost sure valid for all $n \in \mathbb{N}:$

$$
0<a_{n}+\sigma_{n} \xi_{n+1}
$$

Two main results on almost sure asymptotic stability of the solution to (1.2) are obtained. The stability is understood in the standard sense of striving the solution to zero. In the population model (1.2) and similar ones, it means that the population is driven to extinction $[4,5,11]$. The first result is established for the situation when the corresponding deterministic population disappears (the corresponding system without noise is stable). It is shown that the presence of stochastic noise does not change the situation. The second result deals with the case when the corresponding deterministic system is not necessary stable. Then we establish the restrictions on the noise intensity which stabilizes the system. We also obtain a result about the lower limit of the solution to (1.2) for a general noise. The last section contains concluding remarks. Possible generalizations are also discussed.

\section{Preliminary definitions and facts}

Let $\left(\Omega, \mathscr{F},\left\{\mathscr{F}_{n}\right\}_{n \in \mathbb{N}}, \mathbb{P}\right)$ be a complete filtered probability space and $\left\{\xi_{i}\right\}_{i \in \mathbb{N}}$ be a sequence of independent random variables with $\mathbb{E} \xi_{i}=0$. We assume that the filtration $\left\{\mathscr{F}_{n}\right\}_{n \in \mathbb{N}}$ is naturally generated: $\mathscr{F}_{n}=\sigma\left\{\xi_{i}: i=0,1, \ldots, n\right\}$, and use the standard abbreviation "a.s." for the term "almost sure" with respect to the fixed probability measure $\mathbb{P}$.

Among all the sequences $\left\{X_{n}\right\}_{n \in \mathbb{N}}$ of the random variables we distinguish those for which $X_{n}$ are $\mathscr{F}_{n}$-measured for all $n \in \mathbb{N}$.

Definition 2.1. A stochastic sequence $\left\{X_{n}\right\}_{n \in \mathbb{N}}$ is said to be an $\mathscr{F}_{n}$-martingale, if $\mathbb{E}\left|X_{n}\right|<\infty$ and $\mathbb{E}\left(X_{n} \mid \mathscr{F}_{n-1}\right)=X_{n-1}$ a.s. for all $n \in \mathbb{N}$.

Definition 2.2. A stochastic sequence $\left\{X_{n}\right\}_{n \in \mathbb{N}}$ is said to be an $\mathscr{F}_{n}$-submartingale, if $\mathbb{E}\left|X_{n}\right|<\infty$ and $\mathbb{E}\left(X_{n} \mid \mathscr{F}_{n-1}\right) \geq X_{n-1}$ a.s. for all $n \in \mathbb{N}$.

Definition 2.3. A stochastic sequence $\left\{\xi_{n}\right\}_{n \in \mathbb{N}}$ is said to be an $\mathscr{F}_{n}$-martingale-difference, if $\mathbb{E}\left|\xi_{n}\right|<\infty$ and $\mathbb{E}\left(\xi_{n} \mid \mathscr{F}_{n-1}\right)=0$ a.s. for all $n \in \mathbb{N}$. 
The partial sum of martingale differences leads to the following martingale (and conversely):

$$
X_{n}=\sum_{k=1}^{n} \xi_{k} \text { is a martingale } \Longleftrightarrow \xi_{k} \text { is a martingale difference. }
$$

More details on the exploited stochastic notations and concepts can be found in [9, 20]. The following simple but important martingales will be used in this paper.

Example 2.4. Let $\left\{x_{n}\right\}_{n \in \mathbb{N}}$ be a sequence of independent random variables, $\mathbb{E}\left[x_{n}\right]=0$, $\mathbb{E}\left|x_{n}\right|<\infty, \mathscr{F}_{n}=\sigma\left\{\xi_{i}: i=0,1, \ldots, n\right\}$. Let also $\left\{y_{n}\right\}_{n \in \mathbb{N}}, \mathbb{E}\left|y_{n}\right|<\infty$, be a sequence of $\mathscr{F}_{n^{-}}$ measurable random variables. Then

(a) $Z_{n}=\sum_{i=1}^{n} x_{i}$ is an $\mathscr{F}_{n}$-martingale and $x_{n}$ is an $\mathscr{F}_{n}$-martingale difference;

(b) $U_{n}=\sum_{i=1}^{n} y_{i-1} x_{i}$ is an $\mathscr{F}_{n}$-martingale and $y_{n-1} x_{n}$ is an $\mathscr{F}_{n}$-martingale difference.

Definition 2.5. A stochastic sequence $\left\{A_{n}\right\}_{n \in \mathbb{N}}$ is said to be increasing if $A_{n}-A_{n-1} \geq 0$ a.s. for all $n \in \mathbb{N}$.

Lemma 2.6. Let $\left\{X_{n}\right\}_{n \in \mathbb{N}}$ be an increasing stochastic sequence with $\mathbb{E}\left|X_{n}\right|<\infty$ for all $n \in \mathbb{N}$. Then $\left\{X_{n}\right\}_{n \in \mathbb{N}}$ is a submartingale.

The next statement is a version of the Doob decomposition theorem (see, e.g., [20]).

Theorem 2.7. Let $\left\{Y_{n}\right\}_{n \in \mathbb{N}}$ be an $\mathscr{F}_{n}$-submartingale. Then there exist an $\mathscr{F}_{n}$-martingale $\left\{M_{n}\right\}_{n \in \mathbb{N}}$ and an increasing $\mathscr{F}_{n-1}$-measurable stochastic sequence $\left\{A_{n}\right\}_{n \in \mathbb{N}}$ such that a.s. for every $n=1,2, \ldots$, the Doob decomposition takes place:

$$
Y_{n}=M_{n}+A_{n}
$$

Decomposition (2.2) is unique.

The following lemma can be obtained from Theorem 2.7 (see [15] for details).

Lemma 2.8. Let $\left\{\xi_{n}\right\}_{n \in \mathbb{N}}$ be an $\mathscr{F}_{n}$-martingale-difference. Then an $\mathscr{F}_{n}$-martingale-difference $\left\{\mu_{n}\right\}_{n \in \mathbb{N}}$ and a positive $\mathscr{F}_{n-1}$-measurable stochastic sequence $\left\{\eta_{n}\right\}_{n \in \mathbb{N}}$ exist and a.s. for every $n=1,2, \ldots$,

$$
\xi_{n}^{2}=\mu_{n}+\eta_{n}
$$

If $\xi_{n}$ are independent, then for all $n \geq 0$,

$$
\eta_{n}=E\left(\xi_{n}^{2}\right), \quad \mu_{n}=\xi_{n}^{2}-E\left(\xi_{n}^{2}\right) .
$$

To establish the asymptotic stability, we need to apply one of the well-known martingale convergence theorems (e.g., $[9,20])$.

LEMma 2.9. Let $\left\{Y_{n}\right\}_{n \in \mathbb{N}}$ be a nonnegative $\mathscr{F}_{n}$-submartingale, and let

$$
Y_{n}=A_{n}+m_{n}
$$


4 Stability analysis of stochastic Ricker population model

be its Doob decomposition. Then a.s.

$$
\left\{A_{\infty}<\infty\right\} \subseteq\left\{Y_{n} \longrightarrow\right\}
$$

Here by $\left\{Y_{n} \rightarrow\right\}=\left\{\omega \in \Omega: Y_{n}(\omega) \rightarrow\right\}$ we denote the set of all $\omega \in \Omega$ for which $\lim _{t \rightarrow+\infty} Y_{n}(\omega)$ exists and is finite.

The next lemma is a version of the convergence theorem for semimartingale inequalities, which is convenient for this paper.

Lemma 2.10. Let $\left\{Z_{n}\right\}_{n \in \mathbb{N}}$ be a nonnegative $\mathscr{F}_{n}$-measurable process, $\mathbb{E}\left|Z_{n}\right|<\infty$ for all $n \in$ $\mathbb{N}$, and

$$
Z_{n} \leq Z_{n-1}+u_{n}-v_{n}+v_{n}, \quad n=1,2, \ldots,
$$

where $\left\{v_{n}\right\}_{n \in \mathbb{N}}$ is an $\mathscr{F}_{n}$-martingale difference, $\left\{u_{n}\right\}_{n \in \mathbb{N}},\left\{v_{n}\right\}_{n \in \mathbb{N}}$ are nonnegative $\mathscr{F}_{n-1}$ measurable processes, $\mathbb{E}\left|u_{n}\right|, \mathbb{E}\left|v_{n}\right|<\infty$ for all $n \in \mathbb{N}$.

Then

$$
\left\{\sum_{n=1}^{\infty} u_{n}<\infty\right\} \subseteq\left\{\sum_{n=1}^{\infty} v_{n}<\infty\right\} \bigcap\left\{Z_{n} \longrightarrow\right\}
$$

Proof. We have

$$
Z_{n}=Z_{n-1}+u_{n}-v_{n}+v_{n}-\left(Z_{n-1}+u_{n}-v_{n}+v_{n}-Z_{n}\right)=Z_{n-1}+u_{n}-v_{n}+v_{n}-w_{n},
$$

where

$$
w_{n}=Z_{n-1}+u_{n}-v_{n}+v_{n}-Z_{n} \geq 0
$$

is an $\mathscr{F}_{n}$-measurable. Since the sequence $W_{n}=\sum_{i=1}^{n} w_{i}$ is increasing and $\mathscr{F}_{n}$-measurable with $\mathbb{E}\left|W_{n}\right| \leq \sum_{i=1}^{n} \mathbb{E}\left|w_{i}\right|<\infty$ for all $n \in \mathbb{N}$, we conclude from Lemma 2.6 that $\left\{W_{n}\right\}_{n \in \mathbb{N}}$ is an $\mathscr{F}_{n}$-submartingale. Applying Theorem 2.7, we obtain the following representation for $W_{n}$ :

$$
W_{n}=C_{n}+M_{n}^{(1)}
$$

with an $\mathscr{F}_{n}$-martingale $\left\{M_{n}^{(1)}\right\}_{n \in \mathbb{N}}$ and the increasing $\mathscr{F}_{n-1}$-measurable process $\left\{C_{n}\right\}_{n \in \mathbb{N}}$. From the last equality and (2.9), we obtain that

$$
Z_{n}=Z_{0}+U_{n}-\left(V_{n}+C_{n}\right)+\left(M_{n}-M_{n}^{(1)}\right),
$$

where $U_{n}=\sum_{i=1}^{n} u_{i}, V_{n}=\sum_{i=1}^{n} v_{i}, M_{n}=\sum_{i=1}^{n} v_{i}$. The substitution of $\bar{M}_{n}=M_{n}-M_{n}^{(1)}$ and $\bar{U}_{n}=Z_{0}+U_{n}$ into (2.12) for all $n \in \mathbb{N}$ leads to

$$
Z_{n}+\left(V_{n}+C_{n}\right)=\bar{U}_{n}+\bar{M}_{n}=Y_{n}
$$

The stochastic sequence $\left\{Y_{n}\right\}_{n \in \mathbb{N}}$, defined by (2.13), is a nonnegative $\mathscr{F}_{n}$-submartingale with the unique decomposition $Y_{n}=\bar{U}_{n}+\bar{M}_{n}$ into the sum of the $\mathscr{F}_{n}$-martingale 
$\left\{\bar{M}_{n}\right\}_{n \in \mathbb{N}}$ and the $\mathscr{F}_{n-1}$-measurable increasing sequence $\left\{\bar{U}_{n}\right\}_{n \in \mathbb{N}}$. Using Lemma 2.9, we conclude that $a . s$.

$$
\Omega_{1}=\left\{\bar{U}_{\infty}<\infty\right\} \subseteq\left\{Y_{n} \longrightarrow\right\} .
$$

It means that $\lim _{n \rightarrow \infty} Y_{n}$ exists a.s. on $\Omega_{1}$ and therefore $Y_{n}$ is a.s. bounded from above on $\Omega_{1}$. From the left-hand side of (2.13), we have another representation for $Y_{n}$ :

$$
Y_{n}=Z_{n}+\left(V_{n}+C_{n}\right) .
$$

Since $Y_{n}$ is a.s. bounded above on $\Omega_{1}$ and the process $Z_{n}$ is nonnegative, the process $V_{n}+C_{n}$ is also a.s. bounded above on $\Omega_{1}$. Since $V_{n}$ and $C_{n}$ are increasing, there exist the finite limits $\lim _{n \rightarrow \infty} V_{n}$ and $\lim _{n \rightarrow \infty} C_{n}$ a.s. on $\Omega_{1}$. Therefore, the $\operatorname{limit}_{n \rightarrow \infty} Z_{n}$ also exists a.s. on $\Omega_{1}$. The lemma is proven.

\section{Almost sure asymptotic stability of the stochastic Ricker model}

3.1. Case $0<a_{n}<1$. In the case $0<a_{n}<1$, any solution to the deterministic equation (1.1) monotonically tends to zero [11]. Let us are prove that then model (1.2) is also asymptotically stable. Hence, if the dynamic system (1.1) is asymptotically stable, then any noise of the form $e^{-b x_{i}} \sigma_{i} \xi_{i+1}$ cannot change the situation and (1.3) is the only required condition to guarantee the positiveness of the solution (with the initial condition $x_{0}>0$ ).

Theorem 3.1. Suppose that condition (1.3) holds and

$$
\sum_{i=1}^{\infty}\left(a_{i}-1\right)=-\infty, \quad 0<a_{i}<1, \forall i \in \mathbb{N} .
$$

Let $x_{n}$ be a solution to (1.2) with an arbitrary initial value $x_{0}>0$. Then a.s.

$$
\lim _{n \rightarrow \infty} x_{n}=0
$$

Proof. A solution to (1.2) can be represented as

$$
x_{n+1}=x_{0} \prod_{i=0}^{n} e^{-b x_{i}}\left(a_{i}+\sigma_{i} \xi_{i+1}\right) .
$$

Using (1.3), it proves that $x_{n}>0$ for all $n \in \mathbb{N}$ if $x_{0}>0$. The opposite statement is also true: $x_{n}<0$ for all $n \in \mathbb{N}$ if $x_{0}<0$. Equation (1.2) can be rewritten as

$$
x_{n+1}-x_{n}=x_{n}\left(a_{n} e^{-b x_{n}}-1\right)+e^{-b x_{n}} x_{n} \sigma_{n} \xi_{n+1} .
$$

Using the positiveness of $x_{n}$ and $a_{n}$, the difference $x_{n+1}-x_{n}$ can be estimated as

$$
x_{n+1}-x_{n} \leq-x_{n}\left(1-a_{n}\right)+e^{-b x_{n}} x_{n} \sigma_{n} \xi_{n+1}
$$

for all $n \geq 1$. Since $a_{n}<1$ and $x_{n}>0$ for all $n \in \mathbb{N}$, we apply Lemma 2.10 with

$$
Z_{n}=x_{n}, \quad u_{n} \equiv 0, \quad v_{n}=x_{n-1}\left(1-a_{n-1}\right), \quad v_{n} \equiv e^{-b x_{n-1}} x_{n-1} \sigma_{n-1} \xi_{n},
$$


6 Stability analysis of stochastic Ricker population model

and obtain that $\lim _{n \rightarrow \infty} x_{n}$ a.s. exists and a.s.

$$
\sum_{i=1}^{\infty} x_{i}\left(1-a_{i}\right)<\infty
$$

To prove that a.s. $\lim _{n \rightarrow \infty} x_{n}=0$, we assume the opposite: $\lim _{n \rightarrow \infty} x_{n}(\omega) \geq c(\omega)>0$ for $\omega \in \Omega_{1}, \Omega_{1} \subset \Omega, P\left\{\Omega_{1}\right\}>0$. Then, the a.s. finite $N=N(\omega)$ exists such that for all $n \geq N$, and for $\omega \in \Omega_{1}$ we have

$$
\sum_{i=N}^{\infty} x_{i}(\omega)\left(1-a_{i}\right) \geq c(\omega) \sum_{i=N}^{\infty}\left(1-a_{i}\right) \longrightarrow \infty
$$

that contradicts our assumption. Hence, the theorem is proven.

3.2. Case $a_{n} \geq 1$. In the case $a_{n} \geq 1$ the zero equilibrium state of the original deterministic equation is unstable, the population grows and may possess a positive equilibrium state (e.g., $[6,11])$. Let us estimate the lower limit of the solution to the stochastic equation (1.2).

Theorem 3.2. Suppose that condition (1.3) holds, $a_{n} \geq 1$ for all $n>0$, and $\lim _{n \rightarrow \infty} \ln a_{n}$ exists. Let $x_{n}$ be a solution to (1.2) with an arbitrary initial value $x_{0}>0$. Then a.s.

$$
0 \leq \liminf _{n \rightarrow \infty} x_{n} \leq \lim _{n \rightarrow \infty} \frac{\ln a_{n}}{b} .
$$

Proof. Suppose that (3.9) is incorrect, then there exist a set $\Omega_{1} \in \Omega$ and a.s. finite random variables $\delta=\delta(\omega)>0$ and $N=N(\omega)$ such that $\mathbf{P}\left(\Omega_{1}\right)>0$ and for $n \geq N(\omega), \omega \in \Omega_{1}$,

$$
x_{n}(\omega) \geq \frac{\ln a_{n}}{b}+\delta(\omega) .
$$

Then, for any $\omega \in \Omega_{1}$ and $n \geq N(\omega)$, we have

$$
e^{b\left(x_{n}(\omega)-\delta(\omega)\right)} \geq a_{n}, \quad 1-a_{n} e^{-b x_{n}(\omega)} \geq 1-e^{-\delta(\omega) b}=\varepsilon(\omega)>0 .
$$

Since the solution $x_{n}$ to (1.2) is nonnegative, we get

$$
x_{n}(\omega)\left(1-a_{n} e^{-b x_{n}(\omega)}\right) \geq 0
$$

for any $n \geq N(\omega)$ and $\omega \in \Omega_{1}$. Let us take

$$
\chi(u)= \begin{cases}1, & \text { if } u>0 \\ 0, & \text { otherwise }\end{cases}
$$

and rewrite (3.4) as follows:

$$
\begin{aligned}
x_{n+1}-x_{n}= & x_{n}\left(a_{n} e^{-b x_{n}}-1\right) \chi\left[a_{n} e^{-b x_{n}}-1\right] \\
& -x_{n}\left(1-a_{n} e^{-b x_{n}}\right) \chi\left[1-a_{n} e^{-b x_{n}}\right]+e^{-b x_{n}} x_{n} \sigma_{n} \xi_{n+1} .
\end{aligned}
$$


We choose

$$
\begin{gathered}
Z_{n}=x_{n}, \\
u_{n}=x_{n-1}\left(a_{n-1} e^{-b x_{n-1}}-1\right) \chi\left[a_{n-1} e^{-b x_{n-1}}-1\right], \\
v_{n}=x_{n-1}\left(1-a_{n-1} e^{-b x_{n-1}}\right) \chi\left[1-a_{n-1} e^{-b x_{n-1}}\right], \\
v_{n}=e^{-b x_{n-1}} x_{n-1} \sigma_{n-1} \xi_{n} .
\end{gathered}
$$

Noticing that $u_{n} \equiv 0$ on $\Omega_{1}$ for $n \geq N(\omega)+1$, we obtain

$$
\sum_{i=1}^{\infty} u_{i}(\omega)=\sum_{i=1}^{N(\omega)} u_{i}(\omega)<\infty
$$

a.s. on $\Omega_{1}$. Therefore, we can apply Lemma 2.10 and prove that $\lim _{n \rightarrow \infty} x_{n}(\omega)$ exists on $\omega \in \Omega_{1}$ and for $\omega \in \Omega_{1}$,

$$
\begin{aligned}
\infty>\sum_{i=1}^{\infty} v_{i}(\omega) & =\sum_{i=1}^{\infty} x_{i-1}(\omega)\left(1-a_{i-1} e^{-b x_{i-1}(\omega)}\right) \chi\left[1-a_{i-1} e^{-b x_{i-1}(\omega)}\right] \\
& \geq \sum_{i=N(\omega)+1}^{\infty} x_{i-1}(\omega)\left(1-a_{i-1} e^{-b x_{i-1}(\omega)}\right) .
\end{aligned}
$$

However, for $\omega \in \Omega_{1}$,

$$
\begin{aligned}
& \sum_{i=N(\omega)+1}^{\infty} x_{i-1}(\omega)\left(1-a_{i-1} e^{-b x_{i-1}(\omega)}\right) \\
& \quad \geq \varepsilon(\omega) \sum_{i=N(\omega)+1}^{\infty} x_{i-1}(\omega) \geq \varepsilon(\omega) \sum_{i=N(\omega)+1}^{\infty}\left(\frac{\ln a_{i-1}}{b}+\delta(\omega)\right) \geq \varepsilon(\omega) \sum_{i=N(\omega)+1}^{\infty} \delta(\omega)=\infty,
\end{aligned}
$$

that contradicts (3.17). It means that the assumption (3.10) is not correct and (3.9) holds true.

Remark 3.3. At $a_{n} \equiv a>1$, the deterministic model (1.1) has the nontrivial equilibrium state

$$
X^{*}=\frac{\ln a}{b}
$$

Hence, Theorem 3.2 extends the result of Theorem 3.1 in the sense that the demographic stochastic noise cannot improve the population growth of the deterministic Ricker model. Indeed, at any $n$ a solution $x_{n}$ to (1.2) will take the values lower than $X^{*}$ infinite number of times. If the reproduction rate $a_{n}$ tends to its bifurcation value 1 ,

$$
\lim _{n \rightarrow \infty} a_{n}=1
$$


8 Stability analysis of stochastic Ricker population model

we can treat the result of Theorem 3.2 in the following way: there exists the sequence of years $\left\{n_{k}\right\}_{k \in \mathbb{N}}$ such that the population size $x_{n_{k}}$ in these years tends to zero:

$$
\lim _{k \rightarrow \infty} x_{n_{k}}=0
$$

3.3. Case of a large noise. The next theorem explores the case when the noise in (1.2) is large enough and can bring a population to extinction even if the corresponding population without noise does not disappear.

Theorem 3.4. Suppose that there exist $L \in \mathbb{R}$ and $\gamma \in(0,1)$ such that for all $n \in \mathbb{N}$, a.s.

$$
\begin{gathered}
0<a_{n}+\sigma_{n} \xi_{n+1} \leq L, \\
1-a_{n}+\frac{(1-\gamma)}{2(2+L)^{2-\gamma}} \sigma_{n}^{2} \eta_{n} \geq 0, \\
\sum_{i=1}^{\infty}\left(1-a_{i}\right)+\frac{(1-\gamma)}{2(2+L)^{2-\gamma}} \sum_{i=1}^{\infty} \sigma_{i}^{2} \eta_{i}=\infty .
\end{gathered}
$$

Let $x_{n}$ be a solution to (1.2) with an arbitrary initial condition $x_{0}$. Then

$$
\lim _{n \rightarrow \infty} x_{n}=0
$$

Proof. To estimate the difference

$$
x_{n+1}^{\gamma}-x_{n}^{\gamma}=x_{n}^{\gamma} e^{-\gamma b x_{n}}\left(a_{n}+\sigma_{n} \xi_{n+1}\right)^{\gamma}-x_{n}^{\gamma},
$$

we apply the Taylor expansion of the function $y=(1+u)^{\gamma}$ up to the third term

$$
(1+u)^{\gamma}=1+\gamma u+\frac{\gamma(\gamma-1)}{2}(1+\theta)^{\gamma-2} u^{2}
$$

with $\theta$ is between 0 and $\mathrm{u}$. We rewrite

$$
\left(a_{n}+\sigma_{n} \xi_{n+1}\right)^{\gamma}=\left(1+\left[\left(a_{n}-1\right)+\sigma_{n} \xi_{n+1}\right]\right)^{\gamma}
$$

and choose $u=\left(a_{n}-1\right)+\sigma_{n} \xi_{n+1}$. Using (3.22), we obtain that

$$
\begin{gathered}
|u|=\left|\left(a_{n}-1\right)+\sigma_{n} \xi_{n+1}\right| \leq \max \{1, L-1\}, \quad 1+\theta \leq 1+|u| \leq 2+L, \\
\frac{1}{2(1+\theta)^{2-\gamma}} \geq \frac{1}{2(2+L)^{2-\gamma}}, \quad \frac{\gamma(\gamma-1)}{2(1+\theta)^{2-\gamma}} \leq \frac{\gamma(\gamma-1)}{2(2+L)^{2-\gamma}} .
\end{gathered}
$$


Then

$$
\begin{aligned}
x_{n+1}^{\gamma}-x_{n}^{\gamma} & =x_{n}^{\gamma} e^{-\gamma b x_{n}}\left(1+\gamma\left(a_{n}-1\right)+\frac{\gamma(\gamma-1)}{2(2+\theta)^{2-\gamma}}\left[\left(a_{n}-1\right)+\sigma_{n} \xi_{n+1}\right]^{2}\right)-x_{n}^{\gamma} \\
& \leq x_{n}^{\gamma} e^{-\gamma b x_{n}}\left(1+\gamma\left(a_{n}-1\right)+\frac{\gamma(\gamma-1)}{2(2+L)^{2-\gamma}}\left[\left(a_{n}-1\right)+\sigma_{n} \xi_{n+1}\right]^{2}\right)-x_{n}^{\gamma} \\
& =x_{n}^{\gamma} e^{-\gamma b x_{n}}\left(1+\gamma\left(a_{n}-1\right)+\frac{\gamma(\gamma-1)}{2(2+L)^{2-\gamma}}\left[\left(a_{n}-1\right)^{2}+\sigma_{n}^{2} \eta_{n+1}\right]\right)+\tilde{\mu}_{n+1}-x_{n}^{\gamma} \\
& \leq x_{n}^{\gamma} e^{-\gamma b x_{n}}\left(-\gamma\left(1-a_{n}\right)-\frac{\gamma(1-\gamma)}{2(2+L)^{2-\gamma}} \sigma_{n}^{2} \eta_{n+1}\right)+x_{n}^{\gamma}\left(e^{-\gamma b x_{n}}-1\right)+\tilde{\mu}_{n+1} \\
& \leq-x_{n}^{\gamma} e^{\gamma b x_{n}}\left(\gamma\left(1-a_{n}\right)+\frac{\gamma(1-\gamma)}{2(2+L)^{2-\gamma}} \sigma_{n}^{2} \eta_{n+1}\right)+\tilde{\mu}_{n+1},
\end{aligned}
$$

where

$$
\tilde{\mu}_{n+1}=\gamma x_{n}^{\gamma} e^{\gamma b x_{n}}\left(\sigma_{n} \xi_{n+1}+\frac{(\gamma-1)}{2(2+L)^{2-\gamma}}\left[2\left(a_{n}-1\right) \sigma_{n} \xi_{n+1}+\sigma_{n}^{2} \mu_{n+1}\right]\right) .
$$

Then, for all $n \in \mathbb{N}$,

$$
x_{n+1}^{\gamma}-x_{n}^{\gamma} \leq-x_{n}^{\gamma} e^{\gamma b x_{n}}\left(\gamma\left(1-a_{n}\right)+\frac{\gamma(1-\gamma)}{2(2+L)^{2-\gamma}} \sigma_{n}^{2} \eta_{n+1}\right)+\tilde{\mu}_{n+1} .
$$

Since $x_{n}>0$ for all $n \in \mathbb{N}$, the first term in the right-hand side of (3.32) is nonpositive. Hence we can apply Lemma 2.10 to inequality (3.32) with

$$
\begin{gathered}
Z_{n}=x_{n}^{\gamma}, \quad u_{n} \equiv 0, \quad v_{n}=x_{n-1}^{\gamma} e^{\gamma b x_{n-1}}\left(\gamma\left(1-a_{n-1}\right)+\frac{\gamma(1-\gamma)}{2(2+L)^{2-\gamma}} \sigma_{n-1}^{2} \eta_{n}\right), \\
v_{n}=\tilde{\mu}_{n},
\end{gathered}
$$

and obtain that a.s. $\lim _{n \rightarrow \infty} x_{n}$ exists and a.s.

$$
\sum_{i=1}^{n} x_{i}^{\gamma} e^{-\gamma b x_{i}}\left(1-a_{i}+\frac{(1-\gamma)}{2(2+\theta)^{2-\gamma}} \sigma_{i}^{2} \eta_{i+1}\right)<\infty .
$$

To prove that $\lim _{n \rightarrow \infty} x_{n}=0$, we assume the opposite: $\lim _{n \rightarrow \infty} x_{n}(\omega)>0$ for $\omega \in \Omega_{1}, \Omega_{1} \subset$ $\Omega, P\left\{\Omega_{1}\right\}>0$. Then there exist a.s. finite numbers $N=N(\omega)>0$ and $\zeta=\zeta(\omega)>0$ such that for $n \geq N$ and $\omega \in \Omega_{1}$,

$$
x_{n}(\omega) e^{-\gamma b x_{n}(\omega)} \geq \zeta(\omega) .
$$


Applying (3.24), we obtain a contradiction to (3.34): on $\Omega_{1}$,

$$
\begin{aligned}
& \sum_{i=N(\omega)}^{n} x_{i}(\omega) e^{-\gamma b x_{i}(\omega)}\left(\left(1-a_{i}\right)+\frac{(1-\gamma)}{2(2+L)^{2-\gamma}} \sigma_{i}^{2} \eta_{i+1}\right) \\
& \quad \geq \zeta(\omega) \sum_{i=N(\omega)}^{n}\left(\left(1-a_{i}\right)+\frac{(1-\gamma)}{2(2+L)^{2-\gamma}} \sigma_{i}^{2} \eta_{i+1}\right) \longrightarrow \infty, \text { when } n \longrightarrow \infty
\end{aligned}
$$

It proves the theorem.

Remark 3.5. If $a_{n} \geq 1$ and $\lim _{n \rightarrow \infty} a_{n}=1$, then Theorem 3.2 helps to eliminate condition (3.24). Indeed, in this case Theorem 3.2 states that $\liminf _{n \rightarrow \infty} x_{n}=0$. From the proof of Theorem 3.4 it is clear that we need only condition (3.23) for the existence of $\lim _{n \rightarrow \infty} x_{n}$. If it is valid, then $\lim _{n \rightarrow \infty} x_{n}=0$.

In particular, it means that in the case $a_{n} \geq 1, \lim _{n \rightarrow \infty} a_{n}=1$, the intensity of the noise that stabilizes (1.2) can be smaller than in the general case. For example, let us choose

$$
a_{n}=1+\frac{1}{n^{2}}, \quad P\left\{\xi_{n}=1\right\}=\frac{1}{2}, \quad P\left\{\xi_{n}=-1\right\}=\frac{1}{2}, \quad \sigma_{n}=\frac{8}{n},
$$

for all $n \in \mathbb{N}$. Then $\mathbb{E} \xi_{n}=0, \mathbb{E} \xi_{n}^{2}=1$, for all $n \in \mathbb{N}$, and

$$
0<1+\frac{1}{n^{2}}-\frac{8}{n} \leq a_{n}+\sigma_{n} \xi_{n} \leq 1+\frac{1}{n^{2}}+\frac{8}{n} \leq 2
$$

for sufficiently large $n$. Taking $L=2$ and $\gamma=1 / 2$, we prove that condition (3.23) is valid:

$$
1-a_{n}+\frac{(1-\gamma)}{2(2+L)^{2-\gamma}} \sigma_{n}^{2} \eta_{n+1}=-\frac{1}{n^{2}}+\frac{1}{2^{2} 4^{3 / 2}} \frac{2^{6}}{n^{2}}=\frac{1}{n^{2}}>0 .
$$

However, condition (3.24) is not true.

Remark 3.6. Conditions (3.23), (3.24) hold true, in particular, when the reproduction rates $a_{i}$ are small:

$$
a_{i} \leq 1, \forall i \in \mathbb{N}, \quad \sum_{i=1}^{\infty}\left(1-a_{i}\right)=\infty \text {. }
$$

This case reflects a deteriorating deterministic system and is trivial. Then (3.23) and (3.24) are valid for any noise. It means that Theorem 3.1 is a partial case of Theorem 3.4.

If the sign of $a_{i}-1$ is unknown, then the deterministic population with no noise can grow (in particular, when all $a_{n} \geq 1$ [11]). Then Theorem 3.4 can provide the restrictions on the reproduction rates $a_{i}$ and the noise intensities $\sigma_{n}, \eta_{n}$ when the stochastic population does not grow but disappears.

One can see that condition (3.23) is valid for some $\gamma \in(0,1)$ if for all $n \in \mathbb{N}$ a.s.

$$
a_{n} \leq 1+k \sigma_{n}^{2} \eta_{n}=1+\Delta_{n}
$$


where $k<1 /\left(2(2+L)^{2}\right)$. Indeed, since $2 k(2+L)^{2}<1$ and $\lim _{\gamma \rightarrow 0}(1-\gamma)(2+L)^{\gamma}=1$, we can find $\gamma_{0} \in(0,1)$ such that for $\gamma \in\left(0, \gamma_{0}\right)$,

$$
(1-\gamma)(2+L)^{\gamma}>2 k(2+L)^{2} .
$$

Then

$$
1-a_{n}+\frac{(1-\gamma)}{2(2+L)^{2-\gamma}} \sigma_{n}^{2} \eta_{n} \geq-k \sigma_{n}^{2} \eta_{n}+\frac{(1-\gamma)(2+L)^{\gamma}}{2(2+L)^{2}} \sigma_{n}^{2} \eta_{n} \geq 0
$$

If we strengthen condition (3.41) to $a_{n}<1+\Delta_{n}-\varepsilon$ for a small $\varepsilon>0$, then condition (3.24) is also true. Instead of the constant $\varepsilon$, we can take $\varepsilon_{n}=1 / n$ or any other $\varepsilon_{n}$ such that the series $\sum_{n=1}^{\infty} \varepsilon_{n}=\infty$.

Let us consider (3.41). One can see that $\Delta_{n}>0$ for any $a_{n}, \sigma_{n}, \eta_{n}$. Hence, there always exists a domain $\left(1,1+\Delta_{n}\right]$ of the parameter $a_{n}$ such that the population with no noise survives but the stochastically perturbed population extinguishes. More specific conditions for the threshold value $\Delta_{n}$ can be obtained from (3.41) or simulated numerically. In this paper we restrict ourselves with the fundamental fact that such a domain exists and depends on the noise intensities $\eta_{n}$ and $\sigma_{n}$.

In the stationary case $a_{n} \equiv a, \sigma_{n} \equiv \sigma, \eta_{n} \equiv \eta$, the only condition

$$
a<1+\frac{1}{2(2+L)^{2}} \sigma^{2} \eta, \quad \text { where } L=a+\sigma \max _{n \in \mathbb{N}}\left\{\left|\xi_{n+1}\right|\right\}
$$

is enough to satisfy Theorem 3.4.

\section{Concluding remarks}

From the biological point of view, the paper analyzes and clarifies the fundamental fact that certain types of stochastic noise cannot improve the population growth. In fact, it increases the risk of population extinction. We have obtained the exact conditions (3.23) and (3.24) when the demographic-type stochastic noise causes a population to extinguish, which otherwise would exist and grow. These conditions set certain relationships between the population reproduction rates and the intensities of the stochastic noise.

The applied importance of these results depends on the applicability of the Ricker model to real-life biological populations. An important open issue is the color of the stochastic noise experimentally observed in populations. In model (1.2) (as well as in [13 ] and many other investigations), the values in two consecutive steps are assumed to be independent, which results in the "white noise." However, some observations show that the population fluctuations can be positively autocorrelated. Recent results (see [10] and the references therein) have demonstrated that the suggestion of "red" (an autoregressive process) or "pink" environment can either increase or decrease the population extinction risk depending on the model structure.

The authors are going to extend the above results to the equation with multistep delays in the environmental feedback [6]:

$$
x_{n+1}=x_{n} e^{-\sum_{j=0}^{k} b_{j} x_{n-j}}\left(a_{n}+\sigma_{n} \xi_{n+1}\right),
$$


where $k \in \mathbb{N}$ is some fixed number, $b_{j} \geq 0$ for all $i=0,1, \ldots, k$, and $\xi_{n+1}$ are positively autocorrelated, and also to the continuous-time case of Ito type integral equations with distributed delays.

\section{References}

[1] H. Fagerholm and G. Högnäs, Stability classification of a Ricker model with two random parameters, Advances in Applied Probability 34 (2002), no. 1, 112-127.

[2] M. Gyllenberg, G. Högnäs, and T. Koski, Null recurrence in a stochastic Ricker model, Analysis, Algebra, and Computers in Mathematical Research (Luleå, 1992) (M. Gyllenberg and L. Persson, eds.), Lecture Notes in Pure and Appl. Math., vol. 156, Marcel Dekker, New York, 1994, pp. 147164.

[3] , Population models with environmental stochasticity, Journal of Mathematical Biology 32 (1994), no. 2, 93-108.

[4] F. C. Hoppensteadt and C. S. Peskin, Mathematics in Medicine and the Life Sciences, Texts in Applied Mathematics, vol. 10, Springer, New York, 1992.

[5] N. Hritonenko and Y. Yatsenko, Mathematical Modeling in Economics, Ecology and the Environment, Kluwer Academic, Dordrecht, 1999.

[6] _ Applied Mathematical Modelling of Engineering Problems, Applied Optimization, vol. 81, Kluwer Academic, Massachusetts, 2003.

[7] G. Kersting, On recurrence and transience of growth models, Journal of Applied Probability 23 (1986), no. 3, 614-625.

[8] V. Kolmanovskii and L. Shaikhet, General method of Lyapunov functionals construction for stability investigation of stochastic difference equations, Dynamical Systems and Applications, World Sci. Ser. Appl. Anal., vol. 4, World Scientific, New Jersey, 1995, pp. 397-439.

[9] R. Sh. Liptser and A. N. Shiryayev, Theory of Martingales, Mathematics and Its Applications (Soviet Series), vol. 49, Kluwer Academic, Dordrecht, 1989.

[10] J. M. Morales, Viability in a pink environment: why "white noise" models can be dangerous, Ecology Letters 2 (1999), no. 4, 228-232.

[11] J. D. Murray, Mathematical Biology, Biomathematics, vol. 19, Springer, Berlin, 1989.

[12] W. E. Ricker, Stock and recruitment, Journal of Fisheries Research Board of Canada 11 (1954), 559-623.

[13] A. Rodkina, On asymptotic behaviour of solutions of stochastic difference equations, Nonlinear Analysis. Theory, Methods \& Applications. Series A: Theory and Methods 47 (2001), no. 7, 4719-4730, Proceedings of the Third World Congress of Nonlinear Analysts, Part 7 (Catania, 2000).

[14] A. Rodkina and G. Berkolaiko, On asymptotic behavior of solutions to linear discrete stochastic equation, Proceedings of the International Conference "2004-Dynamical Systems and Applications", Antalya, July 2004, pp. 614-623.

[15] A. Rodkina and X. Mao, On boundedness and stability of solutions of nonlinear difference equation with nonmartingale type noise, Journal of Difference Equations and Applications 7 (2001), no. 4, $529-550$.

[16] A. Rodkina, X. Mao, and V. Kolmanovskii, On asymptotic behaviour of solutions of stochastic difference equations with Volterra type main term, Stochastic Analysis and Applications 18 (2000), no. $5,837-857$.

[17] A. Rodkina and H. Schurz, A theorem on global asymptotic stability of solutions to nonlinear stochastic difference equations with Volterra type noises, Stability and Control: Theory and Applications 6 (2004), no. 1, 23-34.

[18] Global asymptotic stability of solutions of cubic stochastic difference equations, Advances in Difference Equations 2004 (2004), no. 3, 249-260. 
[19] _ On global asymptotic stability of solutions of some in-arithmetic-mean-sense monotone stochastic difference equations in $\mathbf{R}^{1}$, International Journal of Numerical Analysis and Modeling 2 (2005), no. 3, 355-366.

[20] A. N. Shiryaev, Probability, 2nd ed., Graduate Texts in Mathematics, vol. 95, Springer, New York, 1996.

Natali Hritonenko: Department of Mathematics, Prairie View A \& M University,

P.O. Box 4189, Prairie View, TX 77446, USA

E-mail address: nahritonenko@pvamu.edu

Alexandra Rodkina: Department of Mathematics and Computer Science, University of the West Indies, Mona, Kingston 7, Jamaica

E-mail address: alexandra.rodkina@uwimona.edu.jm

Yuri Yatsenko: College of Business and Economics, Houston Baptist University, 7502 Fondren Road, Houston, TX 77074, USA

E-mail address: yyatsenko@hbu.edu 


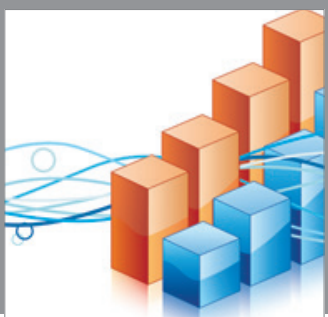

Advances in

Operations Research

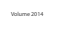

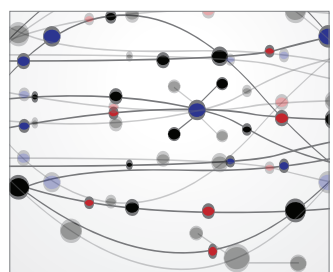

\section{The Scientific} World Journal
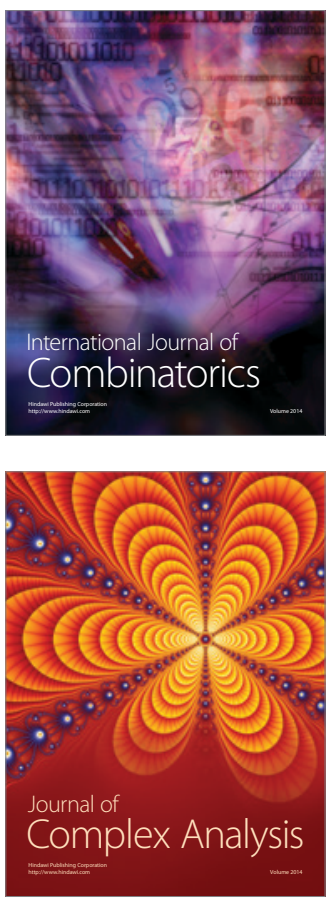

International Journal of

Mathematics and

Mathematical

Sciences
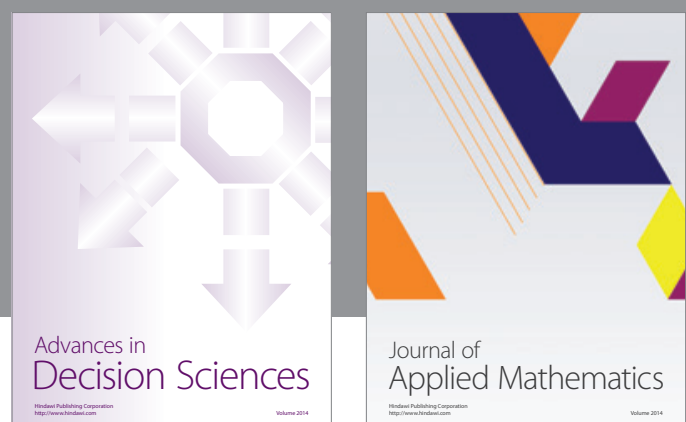

Journal of

Applied Mathematics
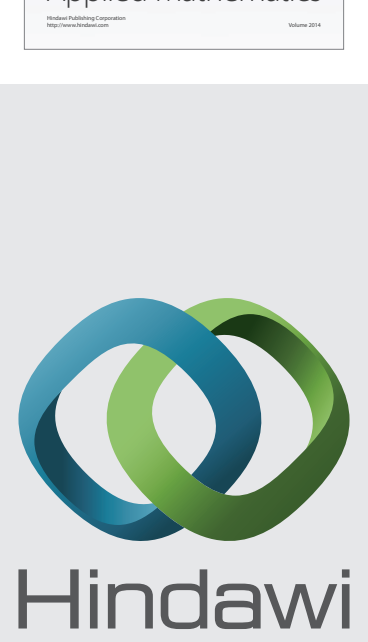

Submit your manuscripts at http://www.hindawi.com
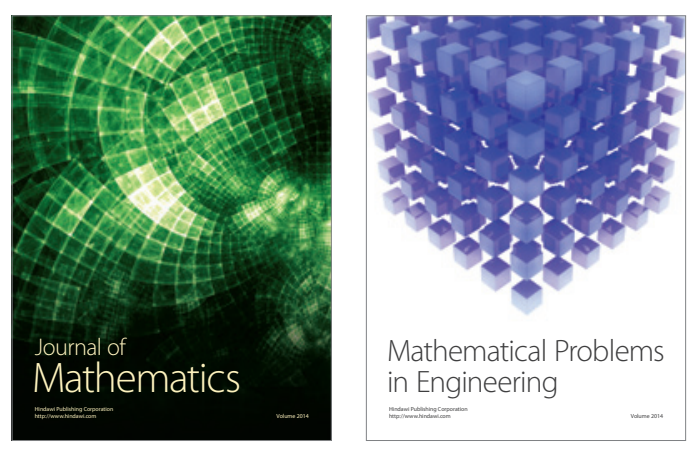

Mathematical Problems in Engineering
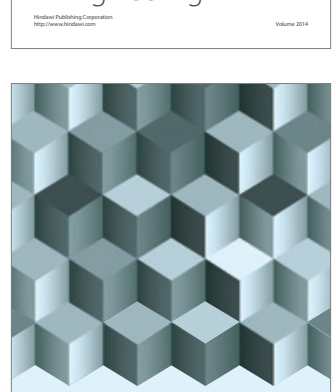

Journal of

Function Spaces
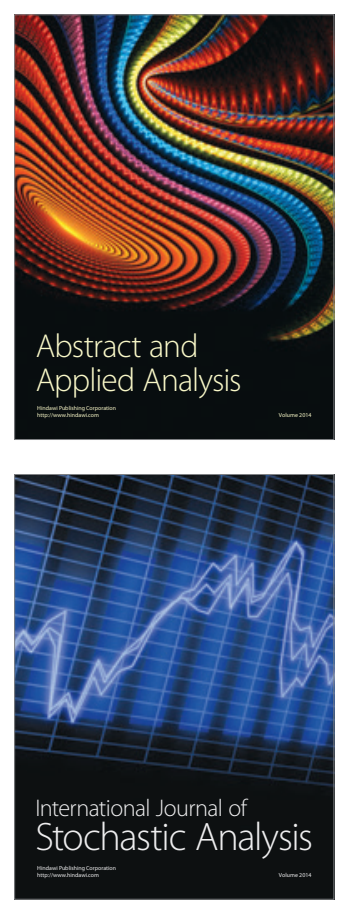

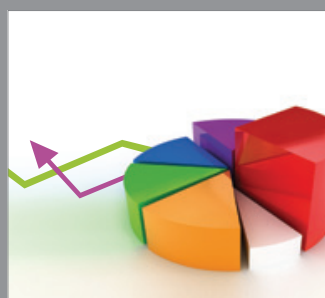

ournal of

Probability and Statistics

Promensencen
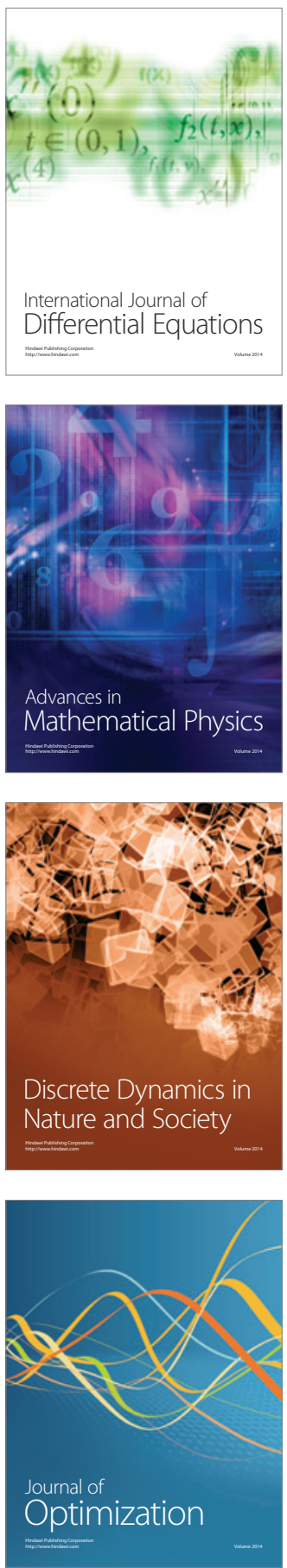